\title{
Prevalence of cardiac myosin-binding protein C3 mutations in Maine Coon cats with hypertrophic cardiomyopathy
}

\author{
Pratch Sukumolanan ${ }^{1}$ (D) and Soontaree Petchdee ${ }^{2}$ iD
}

1. Veterinary Clinical Studies Program, Graduate School, Kasetsart University, Kamphaeng Saen Campus, Nakorn Pathom, 73140, Thailand; 2. Department of Large Animal and Wildlife Clinical Sciences, Faculty of Veterinary Medicine, Kasetsart University, Kamphaeng Saen Campus, Nakorn Pathom, 73140, Thailand.

Corresponding author: Soontaree Petchdee, e-mail: fvetstr@ku.ac.th

Co-author: PS: pratch.vet@gmail.com

Received: 07-10-2021, Accepted: 24-01-2022, Published online: 27-02-2022

doi: www.doi.org/10.14202/vetworld.2022.502-508 How to cite this article: Sukumolanan P, Petchdee S (2022) Prevalence of cardiac myosin-binding protein C3 mutations in Maine Coon cats with hypertrophic cardiomyopathy, Veterinary World, 15(2): 502-508.

\begin{abstract}
Background and Aim: Hypertrophic cardiomyopathy (HCM) is a common heart problem that affects many cats. Although cats with HCM are symptomatic, some die suddenly or develop congestive heart failure. Therefore, this study aimed to estimate the prevalence of myosin-binding protein $\mathrm{C} 3$ (MYBPC3), A31P, and A74T polymorphisms in Maine Coon cats to assess risk factors for diagnosing HCM in cats.
\end{abstract}

Materials and Methods: Forty-nine Maine Coon cats of at least 10 months of age were enrolled in this study. First, clinical parameters, such as heart rate, systolic blood pressure, and echocardiography, were evaluated. Then, polymerase chain reaction, followed by DNA sequencing, was conducted using specific primers for amino acid substitutions caused by genetic variants of $M Y B P C 3-\mathrm{A} 31 \mathrm{P}$ and -A74T polymorphisms.

Results: Investigations showed that the prevalence of $M Y B P C 3-\mathrm{A} 31 \mathrm{P}$ and -A74T mutations in this study was $16.33 \%$ and $24.45 \%$, respectively. Moreover, HCM in cats with MYBPC3-A31P and A74T mutations increased with age, body weight, high heart rate, and prolonged isovolumic relaxation time.

Conclusion: Therefore, we propose that Maine Coon cats develop HCM due to multiple genetic factors and underlying clinical characteristics in individual cats. Furthermore, relaxation time assessments can be a sensitive technique for HCM screening during its preclinical phase and can help identify the risk of developing HCM. However, further studies are warranted to evaluate the effect of $M Y B P C 3$ mutations on the phenotypic expression of HCM.

Keywords: feline, hypertrophic cardiomyopathy, mutation, myosin-binding protein.

\section{Introduction}

Hypertrophic cardiomyopathy (HCM) is an inherited heart problem in the feline population. It is commonly caused by autosomal dominant gene mutations that encode various cardiac sarcomere proteins. Mutations in cardiac myosin-binding protein C3 (MYBPC3), such as A31P and A74T gene polymorphisms, have been proposed to cause HCM in Maine Coon cats [1-3]. Moreover, while the worldwide prevalence of $M Y B P C 3-\mathrm{A} 31 \mathrm{P}$ is approximately $34-42 \%$, the $M Y B P C 3-\mathrm{A} 74 \mathrm{~T}$ mutation is $35 \%$ in Maine Coon and $62 \%$ in other breeds [3-5]. A cohort study revealed that $100 \%$ of cats with homozygous $M Y B P C 3-\mathrm{A} 31 \mathrm{P}$ mutations developed HCM within 5 years of age [6]. Therefore, the American College of Veterinary Internal Medicine (ACVIM) consensus statement guidelines for classifying, diagnosing, and managing cardiomyopathies in cats recently recommended evaluating

Copyright: Sukumolanan and Petchdee. Open Access. This article is distributed under the terms of the Creative Commons Attribution 4.0 International License (http://creativecommons. org/licenses/by/4.0/), which permits unrestricted use, distribution, and reproduction in any medium, provided you give appropriate credit to the original author(s) and the source, provide a link to the Creative Commons license, and indicate if changes were made. The Creative Commons Public Domain Dedication waiver (http:// creativecommons.org/publicdomain/zero/1.0/) applies to the data made available in this article, unless otherwise stated. sarcomeric mutations of $M Y B P C 3$ before breeding to decrease this mutation in the feline population $[7,8]$.

The previous studies have also suggested that the prevalence of HCM was higher in older cats with higher body weights $[9,10]$. Therefore, as a screening method, the feline NT-proBNP plasma concentration can be used to screen for dogs and cats with cardiomyopathy. Furthermore, the atrial natriuretic peptide is proposed to help diagnose HCM in Maine Coon cats as an early screening tool for HCM [11-15]. However, echocardiography is the primary diagnostic tool for $\mathrm{HCM}$ as recommended in the ACVIM guidelines, characterized by increased left ventricular wall thickness. In addition, a recent study demonstrated diastolic myocardial motion using tissue Doppler imaging as an early marker of HCM [16-18]. However, only a few reports on the risk factors and tools for the early detection of HCM in cats exist [19-21].

Therefore, this study aimed to estimate the prevalence of HCM in Maine Coon cats and evaluated risk factors of HCM onset in cats.

\section{Materials and Methods}

\section{Ethical approval and Informed consent}

The study was approved by the Ethical Committee for Animal Experiments, Kasetsart University, 
Thailand (ACKU 62-VET-059). This study involved the use of non-experimental animals only. Verbal consent was obtained from all the owners and no animals or humans are identifiable within this publication,

\section{Study period and location}

This study was conducted from April 2019 to May 2020 at the Kasetsart University Teaching Hospital Kamphaeng Saen, Faculty of Veterinary Science, Kasetsart University.

\section{Animal}

Forty-nine client-owned Maine Coon cats were enrolled in this study. As the inclusion criteria, only cats at least 10 months of age were recruited in this study. However, cats were excluded if they had the following conditions: (1) Secondary left ventricular hypertrophy, such as cardiac structural abnormalities (aortic stenosis), (2) hyperthyroidism (total T4 level $>3.8 \mu \mathrm{g} / \mathrm{dL}$ ), (3) systemic hypertension (systolic blood pressure $>160 \mathrm{mmHg}$ ), (4) pregnancy or were lactating, and (5) previous or current chronic diseases, such as chronic kidney disease, diabetes mellitus, or status epilepticus.

\section{Clinical examination}

Cats were subjected to a complete physical examination to evaluate their general conditions. Hence, 49 Maine Coon cats were enrolled, after which their clinical data, including age, sex, and body weight, were collected. Subsequently, clinical parameters, such as heart rate and blood pressure, were also evaluated. Then, indirect blood pressure measurements using a Doppler device (Parks Medical Electronics, USA) were performed in triplicate, followed by a complete blood count and serum biochemistry profile for each cat as an essential routine health check-up.

\section{DNA sequencing}

With minimal restraints, blood samples (2-3 mL) were collected from the venous vessel of each cat. Then, these samples were stored at $-20^{\circ} \mathrm{C}$ until DNA extraction. Subsequently, nucleic acid was extracted from blood samples $(200 \mu \mathrm{L})$ using a DNA extraction kit (Blood Genomic DNA Extraction Mini Kit, Favorgen, Taiwan) followed by polymerase chain reaction (PCR), using the modified protocol by Godiksen et al. [17]. Briefly, the forward primer was 5'-AGCCTTCAGCAAGAAGCCA-3', and the reverse primer was 5'-CAAACTTGACCTTGGAGGAGC-3'. The PCR process was also conducted using a Thermocycler T-Gradient ThermoBlock (Biometra, Thailand). First, PCR steps were conducted at $95^{\circ} \mathrm{C}$ for $15 \mathrm{~min}, 35$ cycles of $95^{\circ} \mathrm{C}$ for $30 \mathrm{~s}$, $58^{\circ} \mathrm{C}$ for $30 \mathrm{~s}, 72^{\circ} \mathrm{C}$ for $1 \mathrm{~min}$, and final extension at $72^{\circ} \mathrm{C}$ for $10 \mathrm{~min}$. Then, PCR products at $242 \mathrm{bp}$ on a $1.5 \%$ agarose gel electrophoresis were detected and purified using a PCR product purification kit (GEL/ PCR Purification Kit, Favorgen) followed by storage at $-20^{\circ} \mathrm{C}$. Next, Sanger's sequencing was performed to detect the PCR product's nucleotide with a specific forward and reverse primer. Finally, MYBPC3 p.A31P and p.A74T polymorphisms were detected using BioEdit 7.2 (https://bioedit.software.informer.com/) and A plasmid Editor programs (jorgensen.biology. utah.edu/wayned/ape/).

\section{Echocardiography}

Echocardiography (vivid 5s, GE Healthcare, USA) was routinely examined with continuous electrocardiography recording. First, brightness mode (B-mode) and motion mode (M-mode) echocardiography were used to verify the left ventricular structure on the right parasternal short-axis view at the papillary muscle level. The left ventricular hypertrophy was defined as the left ventricular wall with more than $6 \mathrm{~mm}$ thickness [16]. Next, the left atrium and aorta (LA/AO ratio) diameter was determined at the right parasternal short-axis view near the heart base of the AO. Then, the dynamic obstruction at the left ventricular outflow tract was examined. In addition, while the pulse wave Doppler was measured as the $\mathrm{E} / \mathrm{A}$ ratio, isovolumic relaxation time (IVRT) was measured at the left parasternal, apical four-chamber view, as shown in Figure-1.

\section{Statistical analysis}

All values were reported as mean \pm standard error of the mean (SEM). Clinical and echocardiographic parameters were compared between the three groups of no mutation, $M Y B P C 3$ p.A31P mutation, and $M Y B P C 3$ p.A74T mutation through one-way analysis of variance, followed by Tukey's post hoc test using GraphPad Prism 8 (GraphPad Software, San Diego, CA, USA.). Pearson's correlation was then used to test the correlation between age, body weight, and interventricular septum thickness at end-diastole. In addition, odds ratios for developing HCM were generated for positive MYBPC3 p.A31P or p.A74T mutations. Moreover, the efficacy of the diagnostic test was represented by calculating sensitivity and specificity. $\mathrm{p}<0.05$ was considered the minimum level of statistical significance.

\section{Results}

Clinical and echocardiographic characteristics for all cats are reported in Table-1. Results showed that the average age of the cats was $24.72 \pm 3.45$ months, and their average body weight was $6.13 \pm 0.38 \mathrm{~kg}$, of which $23(51.11 \%)$ were male. However, no significant difference was observed in sex or weight between the groups. Results also showed that cats with an A31P mutation were significantly older than those in the A74T mutation group (34.57 vs. 20.44 months, $p=0.0025$ ). Concomitantly, $32.26 \%$ of cats were aged 6-12 months, $45.16 \%$ were aged 13-36 months, and $22.58 \%$ were aged $>36$ months (Figure-2). DNA sequencing results for A31P and A74T mutations in Maine Coon cats are shown in Table-2. Our findings from genetics tests using DNA sequencing indicated that the prevalence of A31P 

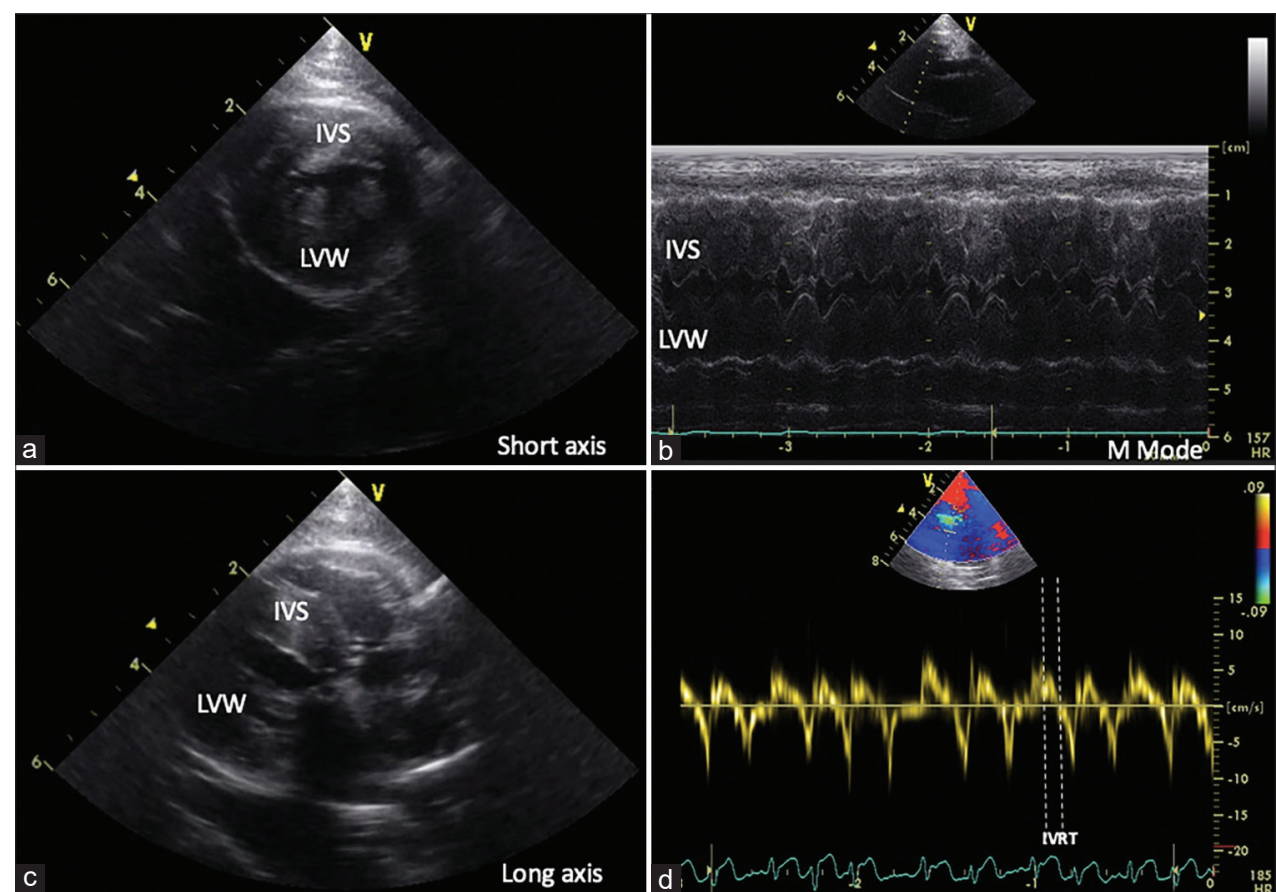

Figure-1: Illustration of echocardiographic recording in HCM affected Maine Coon cats. (a and c) The left ventricular wall is visualized by two-dimensional echocardiography in the short- and long-axis view. (b) Representation of HCM cat; IVS=Interventricular septum, LVW=Left ventricular wall, (d) represented IVRT=Isovolumic relaxation time, $\mathrm{HCM}=$ Hypertrophic cardiomyopathy.

Table-1: Clinical and echocardiographic characteristics of Maine Coon cats (wild type; $\mathrm{n}=33$, and MYBPC3 mutation; $\mathrm{n}=12$ ).

\begin{tabular}{|c|c|c|c|c|}
\hline \multirow[t]{2}{*}{ Clinical parameters } & \multirow[t]{2}{*}{ Wild-type cats } & \multicolumn{2}{|c|}{ Mutation } & \multirow[t]{2}{*}{ p-value } \\
\hline & & A31P & A74T & \\
\hline Age in months & $24.72 \pm 3.45$ & $34.57 \pm 13.07$ & $20.44 \pm 3.39$ & 0.3358 \\
\hline Weight $(\mathrm{kg})$ & $6.13 \pm 0.38$ & $5.75 \pm 2.35$ & $5.41 \pm 0.53$ & 0.9699 \\
\hline Male (number [\%]) & $25(51.02 \%)$ & $50.00 \%$ & $54.54 \%$ & - \\
\hline Heart rate (bpm) & $128.4 \pm 5.44 * *$ & $165.17 \pm 6.74 * *$ & $145.67 \pm 5.01 * *$ & 0.0010 \\
\hline $\mathrm{SBP}(\mathrm{mmHg})$ & $105.5 \pm 9.53$ & $126.25 \pm 6.31$ & $115.5 \pm 1.85$ & 0.2164 \\
\hline \multicolumn{5}{|l|}{ Echocardiography } \\
\hline LA diameter $(\mathrm{cm})$ & $1.19 \pm 0.08$ & $1.31 \pm 0.54$ & $1.38 \pm 0.18$ & 0.9746 \\
\hline LA/AO ratio & $1.42 \pm 0.07$ & $1.23 \pm 0.50$ & $1.28 \pm 0.09$ & 0.6535 \\
\hline IVSd $(\mathrm{cm})$ & $0.63 \pm 0.03$ & $0.56 \pm 0.23$ & $0.56 \pm 0.08$ & 0.6948 \\
\hline LVPWd (cm) & $0.69 \pm 0.04$ & $0.59 \pm 0.24$ & $0.71 \pm 0.10$ & 0.6327 \\
\hline LVIDd $(\mathrm{cm})$ & $1.56 \pm 0.07$ & $1.60 \pm 0.65$ & $1.79 \pm 0.18$ & 0.7562 \\
\hline IVSs $(\mathrm{cm})$ & $0.74 \pm 0.03$ & $0.65 \pm 0.27$ & $0.66 \pm 0.07$ & 0.7144 \\
\hline LVPWs (cm) & $0.78 \pm 0.04$ & $0.67 \pm 0.27$ & $0.71 \pm 0.07$ & 0.4946 \\
\hline LVIDs $(\mathrm{cm})$ & $0.93 \pm 0.06$ & $1.0 \pm 0.40$ & $1.16 \pm 0.13$ & 0.4917 \\
\hline Fractional shortening (\%) & $40.47 \pm 1.68$ & $37.51 \pm 1.53$ & $35.72 \pm 2.42$ & 0.3376 \\
\hline Mitral E/A ratio & $0.96 \pm 0.03$ & $0.92 \pm 0.41$ & $0.93 \pm 0.08$ & 0.9518 \\
\hline IVRT (ms) & $52.09 \pm 2.48 *$ & $72.0 \pm 3.21 *$ & $62.0 \pm 7.07 *$ & 0.0110 \\
\hline
\end{tabular}

Data are presented as mean \pm SEM; $* p<0.05, * * p<0.01$. LA=Left atrium, AO=Aorta, IVSd=Interventricular septal at end-diastole, LVPWd=Left ventricular free proximal wall diameter at end-diastole, LVIDd=Left ventricular internal diameter at end-diastole, IVSs=Interventricular septal at end-systole, LVPWs=Left ventricular free proximal wall diameter at end-systole, LVIDs=Left ventricular internal diameter at end-systole, IVRT=Isovolumic relaxation time

and $\mathrm{A} 74 \mathrm{~T}$ was $16.33 \%$ and $22.45 \%$, respectively. Moreover, the odds ratio of $\mathrm{A} 31 \mathrm{P}$ was 0.72 , and that of A74T was 0.81 .

In addition, echocardiographic results are presented as the mean \pm SEM (Table- 1 ). As observed, no differences existed between cats with the A31P or A74T mutations. Comparison analysis between Maine Coon cats with the $\mathrm{A} 31 \mathrm{P}$ and $\mathrm{A} 74 \mathrm{~T}$ mutations was also performed to corroborate these results. It has been reported that cats with A31P and A74T mutations had a significantly higher heart rate and prolonged IVRT than wild-type cats. However, the results from this study did not significantly differentiate groups based on their systolic blood pressure, left ventricular wall thickness, left atrial diameter, or E/A ratio (Figure-3). Nevertheless, HCM prevalence increased with IVRT for more than $46 \mathrm{~ms}$. In addition, mutation prevalence dramatically increased in cats with a higher level of IVRT having low sensitivity and specificity (Table3). Percentages of age, body weight, and IVRT in 


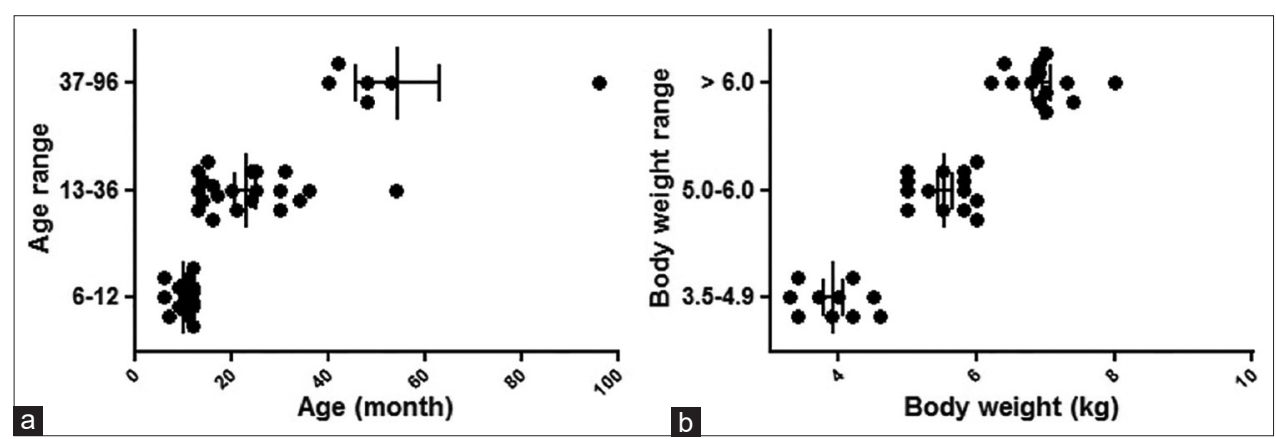

Figure-2: The correlation of age and body weight range of Maine Coon cats. (a) Range of age in Maine Coon cats. (b) Range of body weight in Maine Coon cats. Each dot plot represents individual cats. Pearson's correlation test was performed.
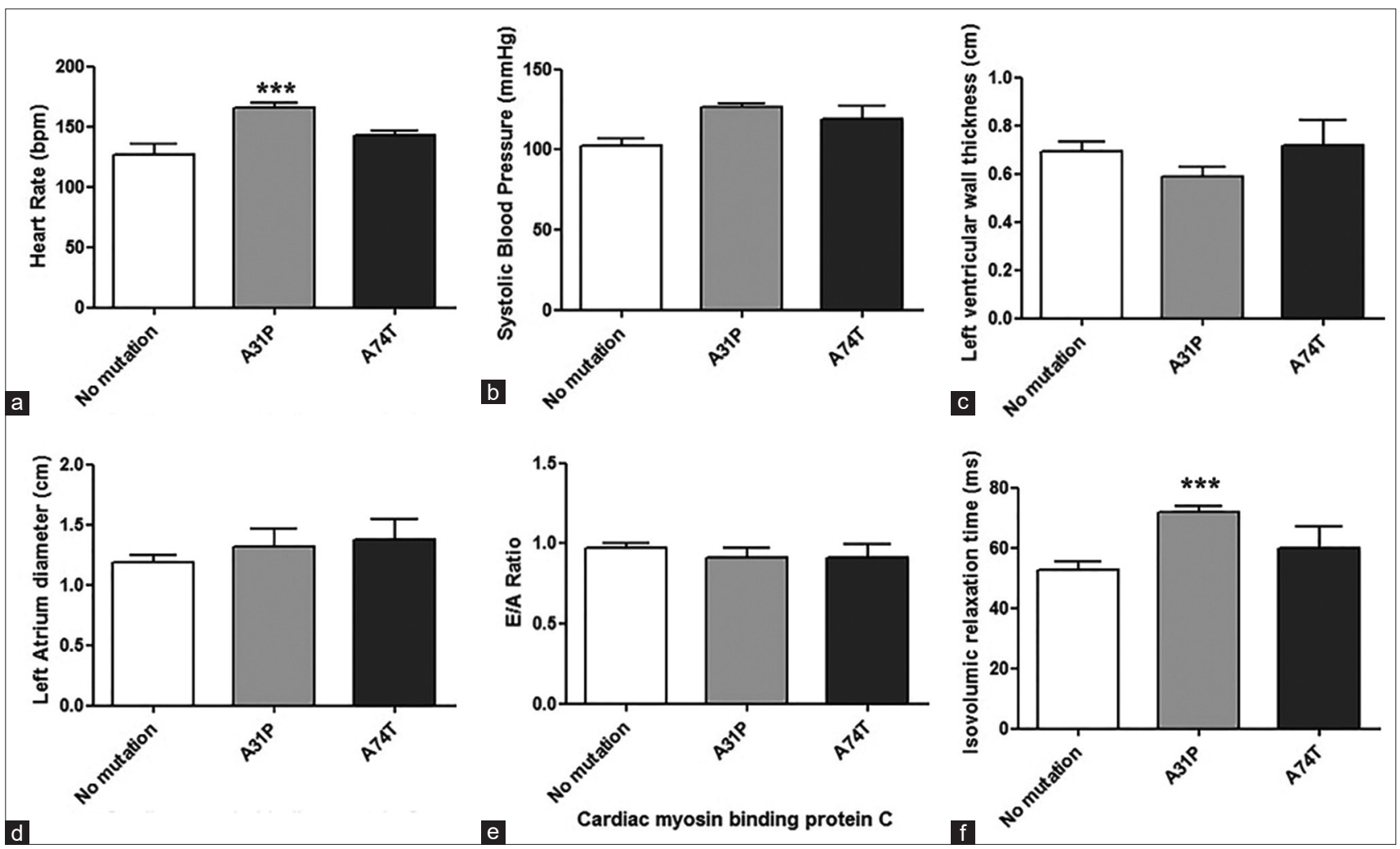

Figure-3: Presentation of clinical and echocardiographic parameters related hypertrophic cardiomyopathy Maine Coon cats. (a) Heart rate (bpm), (b) systolic blood pressure $(\mathrm{mmHg}),(c)$ left ventricular wall thickness (cm), (d) left atrial diameter $(\mathrm{cm}),(e)$ mitral valve E/A ratio, and $(\mathrm{f})$ isovolumic relaxation time $(\mathrm{ms})$ of Maine Coon cats with and without sarcomeric gene mutation. All data expressed as mean \pm SEM. One-way analysis of variants analyzed data with Tukey's multiple comparison test, $* * * \mathrm{p}<0.001$.

Table-2: DNA sequencing results for A31P and A74T SNP in Maine Coon cats.

\begin{tabular}{llcccccc}
\hline SNP & Genotype & \multicolumn{2}{c}{ Phenotype } & n & Prevalence (\%) & OR & 95\%CI \\
\cline { 3 - 6 } & & Normal & Hypertrophic cardiomyopathy & & & \\
\hline A31P & G/G & 28 & 13 & 41 & 16.33 & 0.7179 & $0.1272-4.0507$ \\
& G/C+C/C & 6 & 2 & 8 & 22.45 & 0.8125 & $0.1826-3.6155$ \\
A74T & G/G & 26 & 12 & 38 & 11 & & \\
& G/A+A/A & 8 & 3 & 11 & & \\
\hline
\end{tabular}

$\mathrm{OR}=$ Odds ratio, $\mathrm{G} / \mathrm{G}=$ Homozygous wild type, $\mathrm{G} / \mathrm{C}=$ Heterozygous mutation, $\mathrm{C} / \mathrm{C}=$ Homozygous mutation

HCM Maine Coon cats are presented in Figure-4. The prevalence of HCM was also potentially elevated with increasing age. As observed, although the occurrence of HCM considerably increased between $27.27 \%$ in the juvenile group, $36.84 \%$ in the young group, and $71.43 \%$ in the adult group (Figure-4a), the prevalence of HCM was elevated with increasing body weight (Figure-4b). In contrast, the frequency of HCM using the IVRT parameter fluctuated between the groups (Figure-4c). These results indicated that the presence of prolonged IVRT of more than $46 \mathrm{~ms}$, age between 12 and 96 months, and a body weight of more than $5 \mathrm{~kg}$ were risk factors accounting for HCM (Figures-2 and 4). 


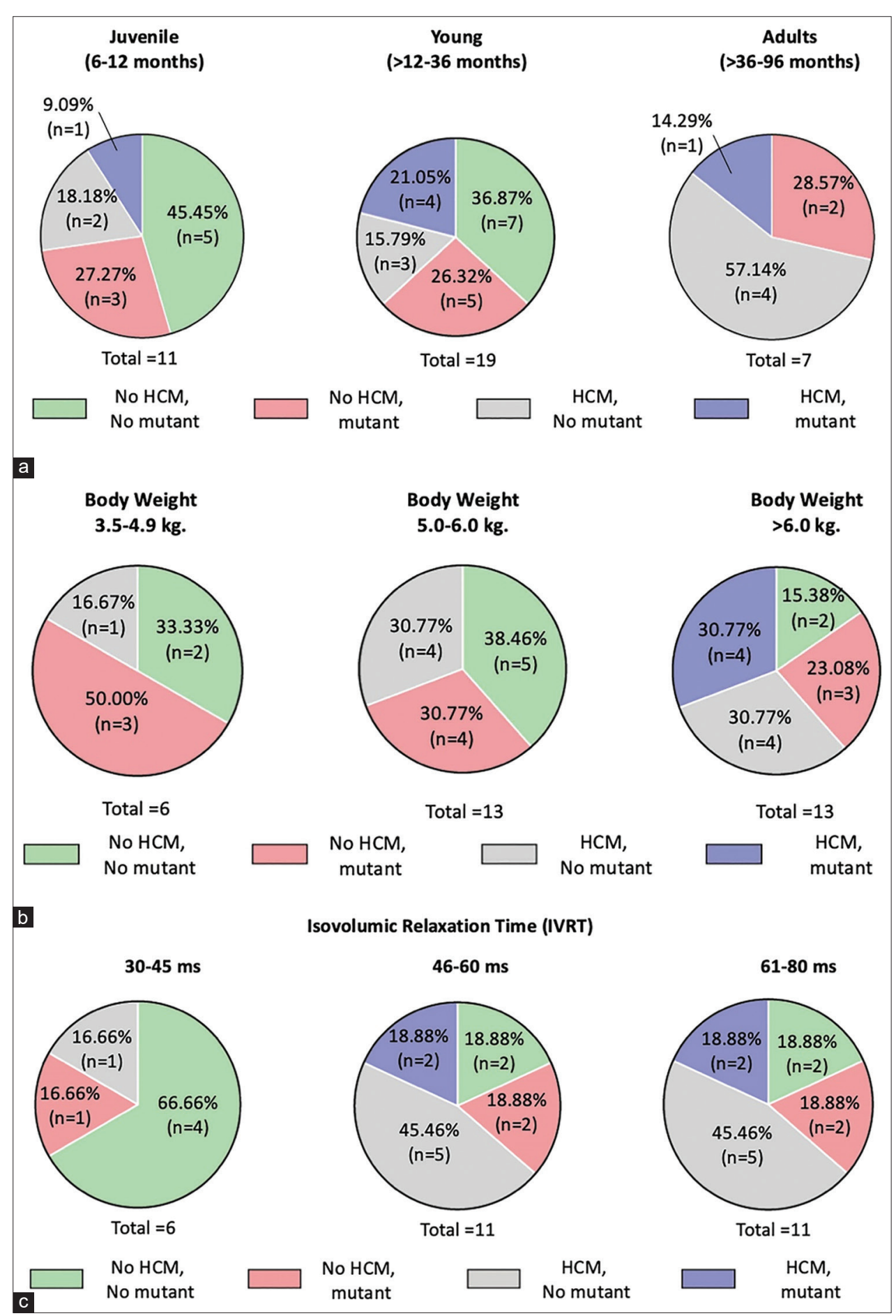

Figure-4: Percentage of age, body weight, and isovolumic relaxation time (IVRT) in hypertrophic cardiomyopathy Maine Coon cats. (a) Age was divided into three ranges of juvenile (6-12 months), young ( $>12-36$ months), and adult ( $>36-96$ months). (b) Body weight was divided into three ranges of $3.5-4.9 \mathrm{~kg}, 5.0-6.0 \mathrm{~kg}$, and $>6.0 \mathrm{~kg}$. (c) IVRT was divided into three ranges of 30-45 $\mathrm{ms}, 46-60 \mathrm{~ms}$, and $61-80 \mathrm{~ms}$.

\section{Discussion}

This study evaluated the prevalence of $M Y B P C 3, \mathrm{~A} 31 \mathrm{P}$, and A74T polymorphisms in Maine Coon cats and investigated risk factors accounting for $\mathrm{HCM}$ in cats. The previous studies have revealed that the prevalence of single-nucleotide polymorphisms in $M Y B P C 3$ was $34-42 \%$ for A31P and $35 \%$ for A74T in Maine Coon cats worldwide [3-5]. In this study, the prevalence of these single-nucleotide polymorphisms in $M Y B P C 3$ was $16.33 \%$ for A31P and $22.45 \%$ for $\mathrm{A} 74 \mathrm{~T}$. However, the odds ratios were 0.72 for $\mathrm{A} 31 \mathrm{P}$ and 0.81 for A74T. Compared with the previous studies $[9,10]$, the prevalence of $M Y B P C 3, \mathrm{~A} 31 \mathrm{P}$, and A74T polymorphisms and the odds ratio was lower in Maine Coon cats with HCM. Furthermore, the occurrence reported in our study was lower than these studies, which can be due to more selective breeding from Maine Coon breeders and convenient genetic testing. However, the HCM prevalence in this study was $30.63 \%$, which was 
Table-3: Isovolumic relaxation time parameter, the prevalence for A31P and A74T mutation, sensitivity and specificity of isovolumic relaxation time to detect HCM in Maine coon cats.

\begin{tabular}{lccc}
\hline Isovolumic relaxation & $\begin{array}{c}\mathbf{3 0 - 4 5} \\
\text { time }(\mathbf{m s})\end{array}$ & $\begin{array}{c}\mathbf{4 6 - 6 0} \\
\mathbf{( \% )}\end{array}$ & $\begin{array}{c}\mathbf{6 1 - 8 0} \\
\mathbf{( \% )}\end{array}$ \\
\hline Mutation prevalence & 14.29 & 36.36 & 50.00 \\
HCM prevalence & 14.29 & 63.63 & 58.33 \\
Sensitivity & 0.00 & 42.86 & 42.86 \\
Specificity & 83.33 & 75.00 & 40.00 \\
\hline
\end{tabular}

HCM=Hypertrophic cardiomyopathy

much higher than that previously reported (14-16\%) $[9,10]$.

According to our data, HCM prevalence with sarcomeric gene mutations increased with age, and an age of more than 12 months was correlated with increased left ventricular wall thickness. This finding agrees with a previous report that increasing age causes thickening of the left ventricle wall in those with an MYBPC3-A31P homozygous mutation [4,6]. Moreover, male cats were more expected to have sarcomeric gene mutations, which had been reported previously [8]. Furthermore, obesity is proposed as the risk factor for HCM in cats [18]. Similarly, this study observed that more than $5 \mathrm{~kg}$ bodyweight was related to the left ventricular wall thickness (Figure-3). In a recent publication, MYBPC3-R820W SNPs were associated with HCM, and body weight was strongly correlated with increased left ventricular proximal wall thickness, left ventricular dimension, and LA/AO ratio. However, no association between sarcomeric gene mutations and left ventricular wall thickness was observed in this study. Previous studies suggested that although the left atrial dilation was a significant risk factor for cardiac death in cats with HCM, the left atrial enlargement can reflect an elevated left ventricular diastolic filling pressure and diastolic dysfunction in HCM patients [20,21]. As observed, the most common factor associated with feline HCM is sudden cardiac death, similar to human HCM. Furthermore, heart functions, such as electrocardiography and echocardiography, are essential diagnostic tools to detect and evaluate many cardiovascular abnormalities. Therefore, a diagnosis would be possible if average values are established for the specific breed [22,23]. Furthermore, in this study, the LA dilation was proposed to be associated with A74T sarcomeric gene mutations (Figure-3). Hence, an $M Y B P C 3-\mathrm{A} 74 \mathrm{~T}$ mutation can reflect another cardiomyopathy phenotype, such as restrictive cardiomyopathy. Nevertheless, a larger sample size in further studies should confirm these results.

Previous evidence has suggested IVRT and the E/A ratio as important echocardiography parameters to indicate the impairment of diastolic functions in cats with HCM that have an MYBPC3-A31P mutation [2]. Besides, studies have reported diastolic myocardial motion as the functional echocardiographic parameter for the early detection of feline HCM [1], similar to our results. Studies have also noted that the low sensitivity of MYBPC3-A31P and A74T testing is a challenge for diagnosing HCM in the feline population.

\section{Conclusion}

It can be concluded that HCM prevalence with sarcomeric gene mutations increased with age, body weight, heart rate, and IVRT. In addition, this study also demonstrated that MYBPC3-A31P and A74T mutations occurred at a high frequency in Maine Coon cats, associated with tachycardia and prolonged relaxation time from echocardiography. Therefore, results proposed that further investigation using diastolic myocardial motion as an early indicator of $\mathrm{HCM}$ in cats and screening $M Y B P C 3$ mutations in cats is needed, especially in Maine Coon cats, as a diagnostic tool for breeding purposes.

\section{Authors' Contributions}

SP: Performed the echocardiography, interpreted the data and results, and manuscript writing and revision. SP and PS: Manuscript writing and figure preparation. Both authors read and approved the final manuscript.

\section{Acknowledgments}

The authors are highly thankful to Kasetsart Veterinary Teaching Hospital and PetD Animal Hospital for providing necessary facilities and support. This study was funded by the National Research Council of Thailand (NRCT): Grant no. NRCT5-RGJ63002-035.

\section{Competing Interests}

The authors declare that they have no competing interests.

\section{Publisher's Note}

Veterinary World remains neutral with regard to jurisdictional claims in published institutional affiliation.

\section{References}

1. Kittleson, M.D., Meurs, K.M., Munro, M.J., Kittleson, J.A., Liu, S.K., Pion, P.D. and Towbin, J.A. (1999) Familial hypertrophic cardiomyopathy in Maine coon cats: An animal model of human disease. Circulation, 99(24): 3172-3180.

2. Meurs, K.M., Sanchez, X., David, R.M., Bowles, N.E., Towbin, J.A., Reiser, P.J., Kittleson, J.A., Munro, M.J., Dryburgh, K., Macdonald, K.A. and Kittleson, M.D. (2005) A cardiac myosin binding protein $\mathrm{C}$ mutation in the Maine Coon cat with familial hypertrophic cardiomyopathy. Hum. Mol. Genet., 14(23): 3587-3593.

3. Wess, G., Schinner, C., Weber, K., Küchenhoff, H. and Hartmann, K. (2010) Association of A31P and A74T polymorphisms in the myosin binding protein $\mathrm{C} 3$ gene and hypertrophic cardiomyopathy in Maine Coon and other breed cats. J. Vet. Intern. Med., 24(3): 527-532.

4. Mary, J., Chetboul, V., Sampedrano, C.C., Abitbol, M., Gouni, V., Trehiou-Sechi, E., Tissier, R., Queney, G., Pouchelon, J.L. and Thomas, A. (2010) Prevalence of the MYBPC3-A31P mutation in a large European feline 
population and association with hypertrophic cardiomyopathy in the Maine Coon breed. J. Vet. Cardiol., 12(3): 155-161.

5. Stern, J.A. and Ueda, Y. (2019) Inherited cardiomyopathies in veterinary medicine. Pflugers Arch., 471(5): 745-753.

6. Granström, S., Godiksen, M.T., Christiansen, M., Pipper, C.B., Martinussen, T., Møgelvang, R., Søgaard, P., Willesen, J.L. and Koch, J. (2015) Genotype-phenotype correlation between the cardiac myosin binding protein $\mathrm{C}$ mutation $\mathrm{A} 31 \mathrm{P}$ and hypertrophic cardiomyopathy in a cohort of Maine Coon cats: A longitudinal study. J. Vet. Cardiol., 17(1): S268-S281.

7. Fuentes, V.L., Abbott, J., Chetboul, V., Côté, E., Fox, P.R., Häggström, J., Kittleson, M.D., Schober, K. and Stern, J.A. (2020) ACVIM consensus statement guidelines for the classification, diagnosis, and management of cardiomyopathies in cats. J. Vet. Intern. Med., 34(3): 1062-1077.

8. Sukumolanan, P., Petchdee, S. (2020) Feline hypertrophic cardiomyopathy: genetics, current diagnosis and management. Vet. Integr. Sci., 18(2): 61-73.

9. Payne, J.R., Brodbelt, D.C. and Fuentes, V.L. (2015) Cardiomyopathy prevalence in 780 apparently healthy cats in rehoming centres (the CatScan study). J. Vet. Cardiol., 17(1): S244-S257.

10. Paige, C.F., Abbott, J.A., Elvinger, F. and Pyle, R.L. (2009) Prevalence of cardiomyopathy in apparently healthy cats. $J$. Am. Vet. Med. Assoc., 234(11): 1398-1403.

11. Laudhittirut, T., Rujivipat, N., Saringkarisate, K., Soponpattana, P., Tunwichai, T. and Surachetpong, S.D. (2020) Accuracy of methods for diagnosing heart diseases in cats. Vet. World, 13(5): 872-878.

12. de Lima, G.V. and da Silveira Ferreira, F. (2017) N-terminalpro brain natriuretic peptides in dogs and cats: A technical and clinical review. Vet. World, 10(9): 1072.

13. Häggström, J., Fuentes, V.L. and Wess, G. (2015) Screening for hypertrophic cardiomyopathy in cats. J. Vet. Cardiol., 17(1): S134-S149.

14. Parzeniecka-Jaworska, M., Garncarz, M. and Kluciński, W. (2016) ProANP as a screening biomarker for hypertrophic cardiomyopathy in Maine coon cats. Pol. J. Vet. Sci., 19(4): 801-807.
15. Jung, S.W., Kittleson, M.D. (2011) The effect of atenolol on NT-proBNP and troponin in asymptomatic cats with severe left ventricular hypertrophy because of hypertrophic cardiomyopathy: A pilot study. J. Vet. Intern. Med., 25(5): 1044-1049.

16. Carlos Sampedrano, C., Chetboul, V., Mary, J., Tissier, R., Abitbol, M., Serres, F., Gouni, V., Thomas, A. and Pouchelon, J.L. (2009) Prospective echocardiographic and tissue Doppler imaging screening of a population of Maine Coon cats tested for the $\mathrm{A} 31 \mathrm{P}$ mutation in the myosin-binding protein $\mathrm{C}$ gene: A specific analysis of the heterozygous status. J. Vet. Intern. Med., 23(1): 91-99.

17. Godiksen, M.T., Granstrøm, S., Koch, J. and Christiansen, M. (2011) Hypertrophic cardiomyopathy in young Maine Coon cats caused by the p. A31P cMyBP-C mutation-the clinical significance of having the mutation. Acta Vet. Scand., 53(1): 1-11.

18. Fox, P.R., Liu, S.K. and Maron, B.J. (1995) Echocardiographic assessment of spontaneously occurring feline hypertrophic cardiomyopathy: An animal model of human disease. Circulation, 92(9): 2645-2651.

19. Freeman, L.M., Rush, J.E., Meurs, K.M., Bulmer, B.J. and Cunningham, S.M. (2013) Body size and metabolic differences in Maine Coon cats with and without hypertrophic cardiomyopathy. J. Feline Med. Surg., 15(2): 74-80.

20. Chetboul, V., Carlos Sampedrano, C., Gouni, V., Nicolle, A.P. and Pouchelon, J.L. (2006) Two-dimensional color tissue Doppler imaging detects myocardial dysfunction before the occurrence of hypertrophy in a young Maine Coon cat. Vet. Radiol. Ultrasound., 47(3): 295-300.

21. Ironside, V.A., Tricklebank, P.R. and Boswood, A. (2021) Risk indicators in cats with preclinical hypertrophic cardiomyopathy: A prospective cohort study. J. Feline Med. Surg., 23(2): 149-159.

22. Sarangi, S., Mahapatra, A.P., Mohapatra, S. and Kundu, A.K. (2016) Age-specific changes in electrocardiographic parameters in bipolar limb leads of conscious female native cats of Odisha. Vet. World, 9(2): 147-150.

23. Brugada-Terradellas, C., Hellemans, A., Brugada, P. and Smets, P. (2021) Sudden cardiac death: A comparative review of humans, dogs, and cats. Vet. J., 274. DOI: 10.1016/j.tvj1.2021.105696

$* * * * * * * *$ 